\title{
Pacific
}

Journal of

Mathematics

\section{PERTURBATIONS OF CERTAIN REFLEXIVE ALGEBRAS}

DAVID RYDER PITTS

Volume $165 \quad$ No. 1 


\title{
PERTURBATIONS OF CERTAIN REFLEXIVE ALGEBRAS
}

\author{
David R. Pitts
}

\begin{abstract}
In this note we use cohomological techniques to prove that if there is a linear map between two CSL algebras which is close to the identity, then the two CSL algebras are similar. We use our result to show that if $\mathscr{L}$ is a purely atomic, hyperreflexive CSL with uniform infinite multiplicity which satisfies the 4-cycle interpolation condition, then there are constants $\delta, C>0$ such that whenever $\mathscr{M}$ is another CSL such that $d(\operatorname{Alg} \mathscr{L}, \operatorname{Alg} \mathscr{M})<\delta$, then there is an invertible operator $S$ such that $S \operatorname{Alg} \mathscr{L} S^{-1}=\operatorname{Alg} \mathscr{M}$ and $\|S\|\left\|S^{-1}\right\|<1+C d(\operatorname{Alg} \mathscr{L}, \operatorname{Alg} \mathscr{M})$.
\end{abstract}

1. Introduction and preliminaries. In this paper, we consider two related types of perturbation questions for CSL algebras. The first deals with the problem of perturbing a linear isomorphism between two algebras to obtain an algebra isomorphism, and the second deals with the problem of deciding whether, for two such algebras, closeness implies isomorphism.

Perturbation questions of this kind were considered by Kadison and Kastler for von Neumann algebras in [18] and further studied by many authors, including Christensen $([\mathbf{3}, \mathbf{4}, \mathbf{5}])$. Johnson ([16]) and Raeburn and Taylor ([21]) obtained results concerning perturbations of closed subalgebras of Banach algebras. Their results show that if $\mathscr{A}$ is a closed subalgebra of a Banach algebra $\mathscr{B}$ and certain cohomology groups for $\mathscr{A}$ vanish, then any closed subalgebra of $\mathscr{B}$ "sufficiently close" to $\mathscr{A}$ is actually isomorphic to $\mathscr{A}$. The nonselfadjoint case was considered first for nest algebras by Lance in [19]. Perturbations of other nonselfadjoint operator algebras were considered by Choi and Davidson ([2]), Davidson ([6]).

In $\S 2$, we prove Theorem 3 which shows that if two CSL algebras $\mathscr{A}_{1}$ and $\mathscr{A}_{2}$ are linearly isomorphic via an isomorphism close to the identity, they they are actually spatially isomorphic via an isomorphism which is close to a unitary equivalence. In $\S 3$, we introduce the 4-cycle interpolation property, which is closely related to a lattice condition appearing in [12] and to the notion of interpolating lattice introduced in [7]. The main result of $\S 3$, Theorem 16, shows that if $\mathscr{A}_{1}$ is a CSL algebra which is sufficiently close to a purely atomic, 
hyperreflexive, infinite multiplicity CSL algebra $\mathscr{A}$ and Lat $\mathscr{A}$ has the 4-cycle interpolation property, then $\mathscr{A}$ and $\mathscr{A}_{1}$ are spatially isomorphic via an isomorphism which is close to the identity. On first sight, it may appear that the assumption that $\mathscr{A}$ is purely atomic might reduce this result to a fairly easy exercise in matching up the atoms of $\operatorname{Lat}(\mathscr{A})$ with those of $\operatorname{Lat}\left(\mathscr{A}_{1}\right)$. However, even in the special case of nest algebras, the theorem is non-trivial. (Recall that a nest algebra is a CSL algebra whose lattice of invariant subspaces is totally ordered.) Indeed the similarity theorem for nest algebras shows that it is entirely possible for a totally atomic nest algebra to be close to a nest algebra which is not totally atomic.

Theorem 16 is closely related to a result obtained by Lance for the nest algebra case. Lance obtained his result by applying the general perturbation results for Banach algebras of Raeburn and Taylor. However, the general Banach algebra results do not apply directly in the CSL setting, for there exist examples of CSL algebras for which the relevant cohomology groups do not vanish. Nevertheless, by using some recent work of Gilfeather and Smith, we are able to obtain our result.

We suspect that Theorem 16 holds in more generality. In particular, it may well be the case that one can relax the hypothesis that one of the algebras be purely atomic to assuming only that the lattice is completely distributive, and it may be possible to adapt our arguments to that case. However, very little concerning hyperreflexivity is known, and it seemed to us that rather than working out the details of a more general argument, it would be better to restrict attention to the simpler, purely atomic case until more is known about hyperreflexivity.

We would like to thank K. Davidson and J. Orr for several useful suggestions, and would particularly like to thank Davidson for pointing out an error in an earlier version of this paper.

Given a subalgebra $\mathscr{A} \subseteq \mathscr{B}(\mathscr{H})$, the lattice of $\mathscr{A}$ is the set of all invariant projections of $\mathscr{A}$ :

$$
\text { Lat } \mathscr{A}=\left\{P \in \operatorname{Proj}(\mathscr{H}): P^{\perp} A P=0 \text { for all } A \in \mathscr{A}\right\} \text {. }
$$

Duality, given a lattice of projections $\mathscr{L}$, the algebra of $\mathscr{L}$ is the set

$$
\operatorname{Alg} \mathscr{L}=\left\{T \in \mathscr{B}(\mathscr{H}): P^{\perp} T P=0 \text { for all } P \in \mathscr{L}\right\} \text {. }
$$

An algebra $\mathscr{A}$ is reflexive if $\mathscr{A}=\operatorname{Alg}$ Lat $\mathscr{A}$, and a subspace lattice $\mathscr{L}$ is reflexive if $\mathscr{L}=\operatorname{Lat} \operatorname{Alg} \mathscr{L}$.

Given a projection lattice $\mathscr{L}$, the diagonal of $\mathscr{L}$ is the algebra

$$
\mathscr{D}(\mathscr{L})=\operatorname{Alg} \mathscr{L} \cap(\operatorname{Alg} \mathscr{L})^{*} \text {. }
$$


Note that $\mathscr{D}(\mathscr{L})$ is the commutant of the set $\mathscr{L}$.

Given a reflexive algebra $\mathscr{A}$ of operators, and $T \in \mathscr{B}(\mathscr{H})$, let

$$
\begin{aligned}
\beta_{\mathscr{A}}(T) & =\sup \left\{\left\|P^{\perp} T P\right\|: P \in \operatorname{Lat}(\mathscr{A})\right\}, \\
k(\mathscr{A}) & =\sup _{T \notin \mathscr{A}} \frac{\|T+\mathscr{A}\|}{\beta_{\mathscr{A}}(T)} .
\end{aligned}
$$

Recall that $\mathscr{A}$ is hyperreflexive if $k(A)<\infty$.

If $P$ and $Q$ are projections on a Hilbert space $\mathscr{H}$, we let $P \vee Q$ denote the projection onto the closed linear span of the ranges of $P$ and $Q$, and $P \wedge Q$ denotes the projection onto the intersection of the ranges of $P$ and $Q$.

A commutative subspace lattice (CSL) is a commuting, strongly closed family $\mathscr{L}$ of projections acting on a separable Hilbert space $\mathscr{H}$ which contains both the identity and the zero operators and which forms a complete lattice under the operations $\vee$ and $\wedge$. Arveson [1] showed that commutative subspace lattices are reflexive. A CSL alge$b r a$ is a reflexive subalgebra of $\mathscr{B}(\mathscr{H})$ which contains a MASA. (The terminology comes from the fact that if $\mathscr{A}$ is a CSL algebra, then Lat $\mathscr{A}$ is a CSL.)

If $\mathscr{L}$ is a CSL and $\mathscr{K}$ is a Hilbert space, we will write

$$
\mathscr{L} \otimes I=\left\{L \otimes I_{\mathscr{K}}: L \in \mathscr{L}\right\} .
$$

We remark that $\operatorname{Alg}(\mathscr{L} \otimes I)$ is the weak-star closure of the span of the elementary tensors $A \otimes B$, where $A \in \operatorname{Alg} \mathscr{L}$ and $B \in \mathscr{B}(\mathscr{H})$.

Recall that the distance $d(V, W)$ between two subspaces $V$ and $W$ of a Banach space $X$ is given by the formula

$$
d(V, W)=\max \left\{\sup _{\substack{v \in V,\|v\|=1}}\|v+W\|, \sup _{\substack{w \in W \\\|w\|=1}}\|w+V\|\right\} .
$$

Recall that two subalgebras $\mathscr{A}_{1}$ and $\mathscr{A}_{2}$ of $\mathscr{B}(\mathscr{H})$ are similar if there exists an invertible operator $T \in \mathscr{B}(\mathscr{H})$ such that $T \mathscr{A}_{1} T^{-1}=$ $\mathscr{A}_{2}$. The class of sub-Boolean operator algebras is the smallest similarity invariant class of subalgebras of $\mathscr{B}(\mathscr{H})$ containing the CSL algebras. Sub-Boolean operator algebras are always reflexive [6].

We shall use the term projection for self-adjoint idempotents in $\mathscr{B}(\mathscr{H})$. If $S \in \mathscr{B}(\mathscr{H})$ is an invertible operator and $P \in \mathscr{B}(\mathscr{H})$ is a projection, $[S P]$ denotes the projection onto the range of $S P$. If $\mathscr{L}$ is a CSL, and $S$ is an invertible operator, then

$$
\text { Lat } S(\operatorname{Alg} \mathscr{L}) S^{-1}=\{[S L]: L \in \mathscr{L}\} \text {. }
$$

We will require the following result of Davidson: 
Proposition 1 ([6], Theorem 2.2). Suppose that $\mathscr{A}_{1}$ and $\mathscr{A}_{2}$ are two sub-Boolean operator algebras with lattices $\mathscr{L}_{1}$ and $\mathscr{L}_{2}$ respectively. Suppose $0<\varepsilon<.01$ and $d\left(\mathscr{A}_{1}, \mathscr{A}_{2}\right)<\varepsilon$. Then there exists a unique complete lattice isomorphism $\theta: \mathscr{L}_{1} \rightarrow \mathscr{L}_{2}$ such that for all $P \in \mathscr{L}_{1}$,

$$
\|P-\theta(P)\|<4 \varepsilon
$$

We shall refer to the lattice isomorphism obtained form Proposition 1 as the standard order isomorphism.

Finally, we shall occasionally refer to the following simple lemma. It is well known and we omit its proof.

LEMMA 2. Suppose that $S \in \mathscr{B}(\mathscr{H})$ is an invertible operator and $\varepsilon>0$. Let $S=W|S|$ be the polar decomposition of $S$ and let $r$ be the square root of $\left\|S^{-1}\right\|\|S\|^{-1}$.

(i) If $U \in \mathscr{B}(\mathscr{H})$ is a unitary operator such that $\|S-U\|<\varepsilon$, then

$$
\|S\|\left\|S^{-1}\right\|<1+\frac{2 \varepsilon}{1-\varepsilon} .
$$

(ii) If $\|S\|\left\|S^{-1}\right\|<1+\varepsilon$, then

$$
\|r S-W\|<\sqrt{1-\varepsilon}-1<\frac{\varepsilon}{2} \text {. }
$$

2. Perturbations of linear isomorphisms. The purpose of this section is to show that if there is a linear map between two CSL algebras which is close to the identity, then the two CSL algebras are similar. More precisely, we will prove the following result:

THeOREM 3. Suppose that $\mathscr{L}$ is a CSL acting on a separable Hilbert space $\mathscr{H}$ and let $i: \operatorname{Alg} \mathscr{L} \rightarrow \mathscr{B}(\mathscr{H})$ be the inclusion map. Then there are constants $0<\delta<.01$ and $C>0$ such that the following statement holds.

Suppose $\mathscr{M}$ is a CSL acting on $\mathscr{H}, \Lambda: \operatorname{Alg} \mathscr{L} \rightarrow \operatorname{Alg} \mathscr{M}$ is a linear isomorphism such that $\|\Lambda-i\|<\delta$, and let $\theta: \mathscr{L} \rightarrow \mathscr{M}$ be the standard order isomorphism. Then there exist an invertible operator $S \in \mathscr{B}(\mathscr{H})$ and a unitary operator $U \in \mathscr{B}(\mathscr{H})$ such that

(i) $\|S-U\|<C\|\Lambda-i\|$ and

(ii) $[S L]=\theta(L)$ for all $L \in \mathscr{L}$. 
We remark that the unitary $U$ appearing in the theorem may be taken to be the identity operator if $\mathscr{L}$ is hyperreflexive. In this case, the constant $C$ will depend also on the hyperreflexivity constant $k(\mathscr{L})$.

We begin by proving several lemmas. The proof of Theorem 3 appears after Lemma 6 below.

In [13], Gilfeather and Moore show that any algebraic isomorphism $\phi$ between two CSL algebras $\mathrm{Alg} \mathscr{L}$ and $\mathrm{Alg} \mathscr{M}$ is automatically continuous and that $\phi$ may be factored as the product of a spatial isomorphism and an automorphism of $\operatorname{Alg} \mathscr{L}$. Our first step towards the proof of Theorem 3 is to show an isomorphism between two CSL algebras which is close to the identity is near a spatial isomorphism which implements the standard order isomorphism.

Lemma 4. Suppose $\mathscr{L}$ and $\mathscr{M}$ are two CSLs acting on the same Hilbert space $\mathscr{H}$. Let $i: \operatorname{Alg} \mathscr{L} \rightarrow \mathscr{B}(\mathscr{H})$ be the inclusion map and suppose $\phi: \operatorname{Alg} \mathscr{L} \rightarrow \operatorname{Alg} \mathscr{M}$ is a Banach algebra isomorphism which satisfies $\|\phi-i\|<.01$ Let $\theta: \mathscr{L} \rightarrow \mathscr{M}$ be the standard lattice isomorphism given by Proposition 1. Then there exist operators $S, U \in \mathscr{B}(\mathscr{H})$ such that:

1. $S$ is an invertible operator and $U$ is a unitary operator;

2. $\|S-U\|<2\|\phi-i\| /(1-2\|\phi-i\|)$; and

3. $[S L]=\theta(L)$ for all $L \in \mathscr{L}$.

Proof. The proof is motivated by the methods of Gilfeather and R. Moore in [13]. For convenience, we let $\varepsilon=\|\phi-i\|$.

Let $\mathscr{C} \subseteq \operatorname{Alg} \mathscr{L}$ be a MASA. Then $\phi(\mathscr{C})$ is a maximal abelian subalgebra of $\mathrm{Alg} \mathscr{M}$. Let $\mathscr{A}$ be the set of all idempotents in $\phi(\mathscr{C})$. Then $\mathscr{A}$ is a Boolean algebra of idempotents and for each $e \in \mathscr{A}$

$$
\|e\| \leq\left\|e-\phi^{-1}(e)\right\|+\left\|\phi^{-1}(e)\right\| \leq \varepsilon+1 .
$$

By a lemma of Wermer in [23], we may find a positive invertible operator $T$ such that $\|I-T\|<2 \varepsilon$ and such that $T e T^{-1}$ is a projection for each $e \in \mathscr{A}$. Let $\mathscr{C}_{1}=T \phi(\mathscr{C}) T^{-1}$. Arguing as Gilfeather and Moore do, we see that $\mathscr{C}_{1}$ is a MASA and $x \mapsto T \phi(x) T^{-1}$ is a *isomorphism between $\mathscr{C}$ and $\mathscr{C}_{1}$. Therefore there exists a unitary $U$ such that

$$
U x U^{*}=T \phi(x) T^{-1} \text { for all } x \in \mathscr{C} .
$$

Now let $S=T^{-1} U$. Then if $L \in \mathscr{L}$, we have $S L S^{-1}=\phi(L)$. It follows that $S \operatorname{Alg} \mathscr{L} S^{-1}=\operatorname{Alg} \mathscr{M}$, and hence $[S L] \in \mathscr{M}$ for each $L \in \mathscr{L}$. 
Note that

$$
\|S-U\|=\left\|T^{-1}-I\right\|<\frac{2 \varepsilon}{1-2 \varepsilon},
$$

and hence items (1) and (2) hold. To verify (3), fix $L \in \mathscr{L}$. We wish to show that

$$
\left\|[S L]-S L S^{-1}\right\|<\sqrt{\varepsilon(2+\varepsilon)} .
$$

Let $z=[S L]\left(S L S^{-1}\right)[S L]^{\perp}$. Since $S L S^{-1}$ and $[S L]$ are idempotents which have the same range, we have

$$
S L S^{-1}=[S L]+z \text {. }
$$

A calculation shows that $\left\|S L S^{-1}\right\|=\sqrt{1+\|z\|^{2}}$ and, therefore,

$$
\|z\|=\sqrt{\left\|S L S^{-1}\right\|^{2}-1} \text {. }
$$

But $\left\|S L S^{-1}\right\|=\|\phi(L)\| \leq 1+\varepsilon$. Inequality 1 now follows. Finally,

$$
\begin{aligned}
\|[S L]-\theta(L)\| & \leq\left\|[S L]-S L S^{-1}\right\|+\|\phi(L)-L\|+\|L-\theta(L)\| \\
& <\sqrt{\varepsilon(2+\varepsilon)}+\varepsilon+4 \varepsilon \\
& <1 \quad(\text { since } \varepsilon<.01) .
\end{aligned}
$$

But $[S L]$ and $\theta(L)$ are commuting projections, so by the last inequality, they must be equal.

This completes the proof.

We now turn our attention to obtaining an algebra isomorphism from a linear isomorphism. We shall be following the strategy used by Lance when considering perturbations of nest algebras in [19]. In this paper, Lance shows that the Hochschild cohomology groups of a nest algebra are trivial, then uses a perturbation result of Raeburn and Taylor from [21]. However, a direct adaptation of Lance's methods to CSL algebras is not possible, since the Hochschild cohomology groups of general CSL algebras need not be trivial (see $[15,14]$ ). Instead, we replace the given CSL algebra with a new CSL algebra which does have trivial cohomology groups, but whose perturbation theory will yield information about perturbations of the original CSL algebra.

Recall from [14] that if $\mathscr{A}$ is a norm closed unital subalgebra of $\mathscr{B}(\mathscr{H})$, then the cone over $\mathscr{A}, \mathscr{C}(\mathscr{A})$, is the algebra

$$
\mathscr{C}(\mathscr{A})=\left\{\left(\begin{array}{ll}
z & 0 \\
u & A
\end{array}\right) \in \mathscr{B}(C \oplus \mathscr{H}): z \in C, u \in \mathscr{H}, A \in \mathscr{A}\right\} .
$$

Also, $\mathscr{E}(\mathscr{A})$ is the algebra

$$
\mathscr{E}(\mathscr{A})=\left\{\left(\begin{array}{ll}
z & u \\
0 & A
\end{array}\right) \in \mathscr{B}(C \oplus \mathscr{H}): z \in C, u \in \mathscr{H}^{*}, A \in \mathscr{A}\right\} .
$$


We remark that if $\mathscr{L}$ is a CSL, then $\mathscr{E}(\mathrm{Alg} \mathscr{L})$ is a CSL algebra and

$$
\text { Lat } \mathscr{E}(\operatorname{Alg} \mathscr{L})=\left\{e_{1} \oplus L: L \in \mathscr{L}\right\} \cup\{0\} \text {; }
$$

here $e_{1}$ is the (rank-one) projection of $C \oplus \mathscr{H}$ onto $C \oplus 0_{\mathscr{H}}$. When $\mathscr{L}$ is a CSL, we shall use the notation

$$
\mathscr{C}(\mathscr{L})=\operatorname{Lat}(\mathscr{C}(\operatorname{Alg} \mathscr{L})) \text { and } \mathscr{E}(\mathscr{L})=\operatorname{Lat}(\mathscr{E}(\operatorname{Alg} \mathscr{L})) \text {. }
$$

Note that if $\mathscr{A}$ is a subalgebra of $\mathscr{B}(\mathscr{H})$ and $\mathscr{A}^{*}$ denotes the algebra whose elements are the adjoints of elements of $\mathscr{A}$, then for each $n \geq 0, H^{n}(\mathscr{A}, \mathscr{B}(\mathscr{H}))$ is isomorphic to $H^{n}\left(\mathscr{A}^{*}, \mathscr{B}(\mathscr{H})\right)$. To see this, define a map $s_{n}: C^{n}(\mathscr{A}, \mathscr{B}(\mathscr{H})) \rightarrow C^{n}\left(\mathscr{A}^{*}, \mathscr{B}(\mathscr{H})\right)$ by

$$
\left(s_{n} \psi\right)\left(A_{1}^{*}, A_{2}^{*}, \ldots, A_{n}^{*}\right)=\left(\psi\left(A_{n}, A_{n-1}, \ldots, A_{1}\right)\right)^{*} \quad\left(A_{j} \in \mathscr{A}\right) .
$$

Then $\partial\left(s_{n} \psi\right)=(-1)^{n+1} s_{n+1}(\partial \psi)$ and hence it follows that $s_{n}$ induces an isomorphism of $H^{n}(\mathscr{A}, \mathscr{B}(\mathscr{H}))$ onto $\mathscr{H}^{n}\left(\mathscr{A}^{*}, \mathscr{B}(\mathscr{H})\right)$.

Gilfeather and Smith prove that $H^{n}(\mathscr{C}(\mathscr{A}), \mathscr{B}(C \oplus \mathscr{H}))=0$ for $n=0,1,2, \ldots$, whenever $\mathscr{A}$ is a norm closed unital subalgebra of $\mathscr{B}(\mathscr{H})$. As $\mathscr{E}(\mathscr{A})=\mathscr{C}\left(\mathscr{A}^{*}\right)^{*}$, the remarks of the preceding paragraph show that

$$
H^{n}(\mathscr{E}(\mathscr{A}), \mathscr{B}(C \oplus \mathscr{H}))=0 \text { for } n=0,1,2, \ldots
$$

To apply the results of Raeburn and Tayor, we need to know that certain cohomology groups of $\mathscr{E}(\mathscr{A})$ with coefficients in $\mathscr{E}(\mathscr{A})$ are trivial. The proof of the following lemma is patterned after an analogous result in [19], and we include an outline for completeness.

Lemma 5. Let $\mathscr{L}$ be a CSL and let $\mathscr{A}=\operatorname{Alg} \mathscr{L}$. Then for $n=$ $1,2,3, \ldots$,

$$
H^{n}(\mathscr{E}(\mathscr{A}), \mathscr{E}(\mathscr{A}))=0 \text {. }
$$

Proof. For ease of notation, let $K=C \oplus \mathscr{H}$.

Let $\sigma \in Z^{n}(\mathscr{E}(\mathscr{A}), \mathscr{E}(\mathscr{A}))$. The fact that the core of $\mathscr{E}(\mathscr{A})$ is an abelian von Neumann algebra enables us to use Theorem 4.1 of [17] to obtain $\phi \in C^{n-1}(\mathscr{E}(\mathscr{A}), \mathscr{E}(\mathscr{A}))$ such that $\rho=\sigma-\partial \phi$ vanishes whenever any of its arguments belong to the core of $\mathscr{E}(\mathscr{A})$. Since $H^{n}(\mathscr{E}(\mathscr{A}), \mathscr{B}(K))=0$, we may find an element

$$
\psi \in C^{n-1}(\mathscr{E}(\mathscr{A}), \mathscr{B}(K))
$$

such that $\partial \psi=\rho$. By Lemma 1.2 of [14], we may assume further that $\psi$ vanishes whenever any of its arguments lie in the core of 
$\mathscr{E}(\mathscr{A})$. The proof of Lemma 2.2 of [19] works for any CSL algebra. Since $\mathscr{E}(\mathscr{A})$ is a CSL algebra, $\psi \in C^{n-1}(\mathscr{E}(\mathscr{A}), \mathscr{E}(\mathscr{A}))$. Thus $\psi+\phi \in C^{n-1}(\mathscr{E}(\mathscr{A}), \mathscr{E}(\mathscr{A}))$ and $\partial \psi+\partial \phi=\rho+\partial \phi=\sigma$. Hence $H^{n}(\mathscr{E}(\mathscr{A}), \mathscr{E}(\mathscr{A}))=0$.

Deleting all appearances of the symbol ' $*$ ' in the first corollary to Theorem 3 of [21] and its proof yields a correct statement and proof. Thus we have

LEMMA 6 [21]. Let $A$ be a closed subalgebra of $\mathscr{B}(\mathscr{H})$ such that $H^{2}(A, A)=H^{3}(A, A)=0$. Then there are constants $\delta>0, C>0$ such that if $B$ is another subalgebra of $\mathscr{B}(\mathscr{H})$ and $\lambda: A \rightarrow B$ is a linear isomorphism satisfying $\|\lambda-i\|<\delta$, where $i: A \rightarrow \mathscr{B}(\mathscr{H})$ is the inclusion, then there is an isomorphism $\phi$ of $A$ onto $B$ with $\|\phi-i\| \leq C\|\lambda-i\|$.

Proof of Theorem 3. Let $\mathscr{L}$ be a CSL acting on $\mathscr{H}$. We may apply Lemma 6 to $\mathscr{E}(\mathrm{Alg} \mathscr{L})$ to obtain constants $\delta_{0}>0, C_{0}>0$ such that if $B$ is a closed subalgebra of $\mathscr{B}(C \oplus \mathscr{H})$ and $\lambda: \mathscr{E}(\operatorname{Alg} \mathscr{L}) \rightarrow B$ is a linear isomorphism satisfying $\|\lambda-i\|<\delta_{0}$, then there exists an algebra isomorphism $\phi: \mathscr{E}(\operatorname{Alg} \mathscr{L}) \rightarrow B$ such that $\|\phi-i\| \leq C_{0}\|\lambda-i\|$.

Let $\delta$ be the smaller of $\delta_{0}$ and $.01 / C_{0}$. Suppose that $\mathscr{M}$ is a CSL acting on $\mathscr{H}$ and that

$$
\Lambda: \operatorname{Alg} \mathscr{L} \rightarrow \operatorname{Alg} \mathscr{M}
$$

is a linear isomorphism which satisfies $\|\Lambda(x)-x\|<\delta\|x\|$ for all $x \in \operatorname{Alg} \mathscr{L}$. Define $\lambda: \mathscr{E}(\operatorname{Alg} \mathscr{L}) \rightarrow \mathscr{E}(\operatorname{Alg} \mathscr{M})$ by

$$
\lambda\left(\left[\begin{array}{ll}
z & u \\
0 & A
\end{array}\right]\right)=\left[\begin{array}{cc}
z & u \\
0 & \Lambda(A)
\end{array}\right] .
$$

Then $\|\lambda(a)-a\|<\delta$ for all $a \in \mathscr{E}(\operatorname{Alg} \mathscr{L})$, so $\|\lambda-i\|<\delta_{0}$. By the remarks above, there exists an algebra isomorphism $\phi: \mathscr{E}(\operatorname{Alg} \mathscr{L}) \rightarrow$ $\mathscr{E}(\mathrm{Alg} \mathscr{M})$ such that

$$
\|\phi(a)-a\| \leq C_{0}\|\lambda(a)-a\| \text { for all } a \in \mathscr{E}(\operatorname{Alg} \mathscr{L}) .
$$

Let $\mathscr{L}^{\prime}=\operatorname{Lat}(\mathscr{E}(\operatorname{Alg} \mathscr{L}))$ and $\mathscr{M}^{\prime}=\operatorname{Lat}(\mathscr{E}(\operatorname{Alg} \mathscr{M}))$. Since $C_{0}\|\lambda-i\|<.01$, Lemma 4 shows that there exist an invertible operator $T \in \mathscr{B}(C \oplus \mathscr{H})$ such that $T \mathscr{L}^{\prime}=\mathscr{M}^{\prime}$ and a unitary operator $V$ in $\mathscr{B}(C \oplus \mathscr{H})$ such that

$$
\|T-V\|<\frac{2 C_{0}\|\lambda-i\|}{1-2 C_{0}\|\lambda-i\|}
$$


By Lemma 2,

$$
\|T\|\left\|T^{-1}\right\|<1+\frac{4 C_{0}\|\lambda-i\|}{1-4 C_{0}\|\lambda-i\|} .
$$

Define an isometry $W: \mathscr{H} \rightarrow C \oplus \mathscr{H}$ by $W u=0 \oplus u$. Set

$$
S_{0}=W^{*} T W \text {. }
$$

By (2), $T$ leaves the subspace $C \oplus 0_{\mathscr{L}}$ invariant. Hence $S_{0}$ is an invertible element of $\mathscr{B}(\mathscr{H})$ and

$$
S_{0}^{-1}=W^{*} T^{-1} W
$$

It follows that the condition number of $S_{0}$ is no greater than the condition number of $T$. Thus if we set

$$
S=\sqrt{\frac{\left\|S_{0}^{-1}\right\|}{\left\|S_{0}\right\|}} S_{0} \text { and } C=\frac{50}{24} C_{0},
$$

then by Lemma 2 there exists a unitary operator $U \in \mathscr{B}(\mathscr{H})$ such that

$$
\|S-U\|<C\|\Lambda-i\|
$$

Next, let $\Theta: \mathscr{L}^{\prime} \rightarrow \mathscr{M}^{\prime}$ be the standard lattice isomorphism and let $K=C \oplus \mathscr{H}$. Since $1 \oplus 0_{\mathscr{H}}$ belongs to $\mathscr{L}^{\prime} \cap \mathscr{M}^{\prime}$, and $\Theta$ is close to the identity, we see that $\Theta\left(1 \oplus 0_{\mathscr{H}}\right)=1 \oplus 0_{\mathscr{H}}$. It follows that if $\theta: \mathscr{L} \rightarrow \mathscr{M}$ is the standard lattice isomorphism, then

$$
\Theta\left(\begin{array}{ll}
1 & 0 \\
0 & L
\end{array}\right)=\left(\begin{array}{cc}
1 & 0 \\
0 & \theta(L)
\end{array}\right)
$$

Thus, for $L \in \mathscr{L}$,

$$
\begin{aligned}
S L \mathscr{H} & =W^{*} T(0 \oplus L) K \subseteq W^{*} T(I \oplus L) K \\
& =W^{*} \Theta(I \oplus L) K=W^{*}(I \oplus \theta(L)) K \\
& =\theta(L) \mathscr{H} .
\end{aligned}
$$

A similar argument using $S^{-1}$ and interchanging the roles of $\mathscr{L}$ and $\mathscr{M}$ yields that $S^{-1} \theta(L) \mathscr{H} \subseteq L \mathscr{H}$. Therefore,

$$
[S L]=\theta(L) \text { for all } L \in \mathscr{L} \text {. }
$$

3. Perturbations of certain CSL algebras. We begin this section with two definitions. The first is a property which a CSL algebra may possess, while the second is a lattice condition.

Definition 7. Say that a CSL algebra $\operatorname{Alg} \mathscr{L}$ acting on a Hilbert space $\mathscr{H}$ has the algebra perturbation property if there exist positive constants $C$ and $\delta<.01$ such that the following statement holds: 
'If $\mathscr{M}$ is a commutative subspace lattice acting on $\mathscr{H}$ such that $d(\operatorname{Alg} \mathscr{L}, \operatorname{Alg} \mathscr{M})<\delta$ and $\theta: \mathscr{L} \rightarrow \mathscr{M}$ is the standard order isomorphism, there exist an invertible operator $S \in \mathscr{B}(\mathscr{H})$ and a unitary operator $U \in \mathscr{B}(\mathscr{H})$ such that

(i) $\|S-U\|<C d(\operatorname{Alg} \mathscr{L}, \operatorname{Alg} \mathscr{M})$ and

(ii) $[S L]=\theta(L)$ for all $L \in \mathscr{L}$.'

Recall that if $\mathscr{L}$ is a CSL, an interval of $\mathscr{L}$ is a projection of the form $L_{1} L_{2}^{\frac{1}{2}}$ for some projections $L_{1}, L_{2} \in \mathscr{L}$. It is easy to see that any atom of $\mathscr{L}$ is an interval. Given disjoint intervals $E$ and $F$ of $\mathscr{L}$, we write $E \preceq \mathscr{L} F$ if $E \operatorname{Alg} \mathscr{L} F=E \mathscr{B}(\mathscr{H}) F$. If no confusion can arise, we will simply write $\preceq$ instead of $\preceq \mathscr{L}$.

We shall require a notion concerning interpolation of 4-cycles in lattices which appears in the characterization of tree algebras in [12] and is also one of the two conditions required for a CSL to be an interpolating lattice in the sense of Davidson (cf. [7], Definition 5.1).

Definition 8. Let us say that a CSL has the 4-cycle interpolation property if whenever $A_{i}, B_{i}(i=1,2)$ are non-zero disjoint intervals of $\mathscr{L}$ such that $B_{1} \preceq A_{1} \succeq B_{2} \preceq A_{2} \succeq B_{1}$ is a 4-cycle, there are non-zero subintervals $A_{i}^{\prime} \leq A_{i}$ and $B_{i}^{\prime} \leq B_{i}$ and a non-zero interval $G$ of $\mathscr{L}$ such that $B_{i}^{\prime} \preceq G \preceq A_{i}^{\prime}$ for $i=1,2$.

Of course, if $A_{i}$ and $B_{i}$ are atoms of $\mathscr{L}$, then $A_{i}^{\prime}=A_{i}$ and $B_{i}^{\prime}=B_{i}$.

In this section we apply Theorem 3 to show that a CSL algebra whose lattice $\mathscr{L}$ is hyperreflexive, infinite multiplicity, purely atomic and satisfies the 4-cycle interpolation condition has the algebra perturbation property. This result is Theorem 16 below. We suspect that the condition that $\mathscr{L}$ is purely atomic can be weakened to requiring that $\mathscr{L}$ be completely distributive, but we have not checked carefully. While none of these conditions are necessary conditions, our result does broaden the class of algebras known to have nice perturbation properties.

Our basic strategy will be to use Proposition 10 below to show that under certain circumstances, two close hyperreflexive CSL algebras are similar.

The following lemma is Proposition A of [20]:

LEMMA 9 ([20]). Suppose $\mathscr{L}$ is a hyperreflexive CSL and $0<\varepsilon<$ $1 / 4$. If $U$ is a unitary operator such that

$$
\sup _{L \in \mathscr{L}}\left\|U L U^{*}-L\right\|<\frac{\varepsilon}{k(\mathscr{L})}
$$


then there is an invertible operator $T \in \mathscr{B}(\mathscr{H})$ with $\|T-I\|<\varepsilon$ and such that $U L U^{*}=[T L]$ for each $L \in \mathscr{L}$.

Proposition 10. Suppose that $\mathscr{L}$ is a CSL and that there exists an increasing net $\left\{F_{\lambda}\right\}_{\lambda \in \Lambda}$ of hyperreflexive sublattices whose union is strongly dense in $\mathscr{L}$ and such that $\sup _{\lambda} k\left(\mathscr{F}_{\lambda}\right)=M<\infty$. Then $\mathrm{Alg} \mathscr{L}$ has the algebra perturbation property.

Proof. Let $\delta_{0}>0$ and $C>0$ be the constants associated to $\operatorname{Alg} \mathscr{L}$ by Theorem 3 and let $\delta=\delta_{0} / 4 M$.

Suppose that $\mathscr{M}$ is a CSL such that $d(\operatorname{Alg} \mathscr{L}, \operatorname{Alg} \mathscr{M})<\delta$ and set

$$
\eta=d(\operatorname{Alg} \mathscr{L}, \operatorname{Alg} \mathscr{M}) .
$$

Let $\theta: \mathscr{L} \rightarrow \mathscr{M}$ be the standard order isomorphism. Then by Proposition 1 , we have

$$
\|L-\theta(L)\| \leq 4 \eta \text { for all } L \in \mathscr{L} .
$$

For $\lambda \in \Lambda$, let $G_{\lambda}=\theta\left(\mathscr{F}_{\lambda}\right)$.

Each atom of $\mathscr{F}_{\lambda}$ is a difference of two projections of $\mathscr{F}_{\lambda}$ and hence $\theta$ induces a map, which we shall also denote by $\theta$, between the atoms of $\mathscr{F}_{\lambda}$ and the atoms of $G_{\lambda}$ such that

$$
\|A-\theta(A)\| \leq 8 \eta<8 \delta<1
$$

for each atom $A$ of $\mathscr{F}_{\lambda}$. Hence there exists a partial isometry $u_{A}$ with initial space $A$ and range space $\theta(A)$. If $U_{\lambda}=\sum u_{A}$, where the sum is over all the atoms of $\mathscr{F}_{\lambda}$, then $U_{\lambda}$ is a unitary operator such that

$$
U_{\lambda} L U_{\lambda}^{*}=\theta(L) \text { for each } L \in \mathscr{L}_{\lambda} .
$$

By (3) and Lemma 9, there is an invertible operator $T_{\lambda} \in \mathscr{B}(\mathscr{H})$ such that

$$
\left\|T_{\lambda}-I\right\|<4 \eta M<\delta_{0}
$$

and

$$
\left[T_{\lambda} L\right]=\theta(L) \text { for all } L \in \mathscr{F}_{\lambda} \text {. }
$$

Arguing as Lance does in the proof of Lemma 4.6 of [19], we conclude that there exists an invertible linear map $\Phi: \mathscr{B}(\mathscr{H}) \rightarrow \mathscr{B}(\mathscr{H})$ such that

$$
\left\|\Phi-\operatorname{Id}_{\mathscr{B}(\mathscr{H})}\right\|<\delta_{0}
$$

and the restriction of $\Phi$ to $\operatorname{Alg} \mathscr{L}$ is a linear isomorphism of $\operatorname{Alg} \mathscr{L}$ into $\mathrm{Alg} \mathscr{M}$. An application of Theorem 3 now completes the proof. 
Question. The hypotheses on $\mathscr{L}$ in Proposition 10 imply that $\mathscr{L}$ is hyperreflexive and that $k(\mathscr{L}) \leq M$. The question then arises: Does $a$ hyperreflexive CSL $\mathscr{L}$ always have such a net? Unfortunately, hyperreflexivity behaves badly when passing to sublattices. Indeed, Davidson and Power in [10] show that finite sublattices of a complemented atomic lattice may have arbitrarily large distance constants. However, it follows from a result of Rosenoer [22] that all complemented CSLs are hyperreflexive.

We would like to have an affirmative answer to this question, for then all hyperreflexive CSLs would have the perturbation property.

One way to construct CSLs with well behaved distance constants is to look at compressions to diagonal projections. For if $R$ is a projection in $\mathscr{D}(\mathscr{L})$, then

$$
k(R \mathscr{L}) \leq k(\mathscr{L}) .
$$

To see this, choose $T \in \mathscr{B}(\mathscr{H})$, and view $R T R$ as an operator on the range of $R$. We have

$$
\begin{aligned}
d(R T R, \operatorname{Alg} R \mathscr{L}) & =\inf _{A \in \operatorname{Alg} \mathscr{L}}\|R T R-R A R\| \\
& \leq d(R T R, \operatorname{Alg} \mathscr{L}) \\
& \leq k(\mathscr{L}) \beta_{\mathscr{L}}(R L R) \\
& =k(\mathscr{L}) \mathscr{B}_{r \mathscr{L}}(R L R) ;
\end{aligned}
$$

the last equality follows from the fact that $R$ commutes with $\mathscr{L}$. Thus (4) holds.

Hence if there exists a finite sublattice $\mathscr{F}$ of $\mathscr{L}$ which is order isomorphic to $R \mathscr{L}$, one would obtain a bound on the distance constant of $\mathscr{F}$ in terms of $k(\mathscr{L} \otimes I)$. The reason we do not obtain a bound in terms of $k(\mathscr{L})$ is that we do not have any information on the relationship between the multiplicities of $R \mathscr{L}$ and $\mathscr{F}$. This would make no difference for our purposes if we were able to show the existence of a positive constant $c$ such that $k(\mathscr{L} \otimes I) \leq c k(\mathscr{L})$. (The inequality (4) implies that $k(\mathscr{L}) \leq k(\mathscr{L} \otimes I)$.) We should mention that the problem of understanding the relationship between the distance constants of $\mathscr{L}$ and $\mathscr{L} \otimes I$ also arose in the recent paper of Davidson and Ordower [8].

Let us say that an atomic CSL $\mathscr{L}$ has the compression property if there exists a net $\left\{R_{\lambda}\right\}_{\lambda \in \Lambda}$ in the commutant of $\mathscr{L}$ and finite sublattices $\left\{\mathscr{F}_{\lambda}\right\}$ of $\mathscr{L}$ such that:

1. $\bigcup_{\lambda} \mathscr{F}_{\lambda}$ is strongly dense in $\mathscr{L}$, 
2. $R_{\lambda} \mathscr{L}=R_{\lambda} \mathscr{F}_{\lambda}$ for all $\lambda \in \Lambda$, and

3. $\mathscr{F}_{\lambda}$ is order isomorphic to either $R_{\lambda} \mathscr{L}$ or to $\mathscr{C}\left(R_{\lambda} \mathscr{L}\right)$.

In this definition, we would have preferred the simpler condition that $\mathscr{F}_{\lambda}$ is order isomorphic to $R_{\lambda} \mathscr{L}$. However this is too strong a condition for our purposes, for we will want a purely atomic CSL $\mathscr{L}$ for which the identity is a strong limit point to enjoy the compression condition provided it also has the 4-cycle interpolation property.

The compression condition is enough to ensure that infinite multiplicity lattices satisfy the hypotheses of Proposition 10.

Proposition 11. Suppose $\mathscr{L}$ is a hyperreflexive, infinite multiplicity CSL which has the compression property. Then $\mathrm{Alg} \mathscr{L}$ has the perturbation property.

Proof. Suppose that $R_{\lambda}$ and $\mathscr{F}_{\lambda}$ are as in the definition of the compression property. We shall show that for all $\lambda$,

$$
k\left(\mathscr{F}_{\lambda}\right) \leq k(\mathscr{L})+1
$$

The proposition will then follow from Proposition 10.

Since $\mathscr{L}$ has uniform infinite multiplicity, so does $\mathscr{F}_{\lambda}$. If $\mathscr{F}_{\lambda}$ is order isomorphic to $R_{\lambda} \mathscr{L}$, we have

$$
k\left(\mathscr{F}_{k}\right)=k\left(\left(R_{\lambda} \mathscr{L}\right) \otimes I\right) \leq k(\mathscr{L}),
$$

and all is well.

On the other hand, if $\mathscr{F}_{\lambda}$ is order isomorphic to $\mathscr{C}\left(R_{\lambda} \mathscr{L}\right)$, let $G_{\lambda}$ be an infinite multiplicity CSL which is order isomorphic to $R_{\lambda} \mathscr{L}$. As above, we have

$$
k\left(G_{\lambda}\right)=k\left(\left(R_{\lambda} \mathscr{L}\right) \otimes I\right) \leq k(\mathscr{L}) .
$$

Then $\operatorname{Alg} \mathscr{F}_{\lambda}$ is unitarily equivalent to the algebra of all $2 \times 2$ operator matrices $\left(T_{i j}\right)$ such that $T_{i 1} \in \mathscr{B}(\mathscr{H})(i=1,2), T_{12}=0$ and $T_{22} \in$ $\operatorname{Alg} G_{\lambda}$. Thus $\mathscr{F}_{\lambda}$ is unitarily equivalent to $\left\{0 \oplus P: P \in G_{\lambda}\right\} \cup\{I \oplus I\}$. Identify $\mathscr{F}_{\lambda}$ with its image under this unitary equivalence. If $S=$ $\left(S_{i j}\right) \in \mathscr{B}(\mathscr{H} \oplus \mathscr{H})$, one has

$$
d\left(S, \operatorname{Alg} \mathscr{F}_{\lambda}\right)=\inf _{A_{22} \in \operatorname{Alg} G_{\lambda}}\left\|\left(\begin{array}{c}
S_{12} \\
S_{22}-A_{22}
\end{array}\right)\right\| \leq d\left(S_{22}, \operatorname{Alg} G_{\lambda}\right)+\left\|S_{12}\right\|
$$

and

$$
\beta_{\mathscr{F}}(S)=\sup _{P \in G_{\lambda}}\left\|\left(\begin{array}{c}
S_{12} \\
P^{\perp} S_{22} P
\end{array}\right)\right\| \geq \max \left\{\left\|S_{12}\right\|, \beta_{G_{\lambda}}\left(S_{22}\right)\right\} .
$$


It follows that

$$
\frac{d\left(S, \operatorname{Alg} \mathscr{F}_{\lambda}\right)}{\beta_{\mathscr{F}}(S)} \leq k(\mathscr{L})+1
$$

We conclude that (5) holds in this case as well. This completes the proof.

Our goal is to show that purely atomic CSLs which have the 4-cycle interpolation property also enjoy the compression property. We shall require several lemmas.

If $R$ is an interval of a CSL $\mathscr{L}$, let

$$
L(R)=\bigwedge\{L \in \mathscr{L}: R \leq L\} .
$$

The following is related to Lemma 5.6 of [7].

Lemma 12. Suppose $\mathscr{L}$ is a purely atomic CSL which has the 4cycle interpolation property, that $k \geq 1$ is an integer, and that $A_{1}, \ldots$, $A_{k}$ are distinct atoms of $\mathscr{L}$ such that

$$
P=\left(\bigwedge_{i=1}^{k} L\left(A_{i}\right)\right) \neq 0 .
$$

If $B_{1}$ and $B_{2}$ are atoms of $\mathscr{L}$ such that $B_{i} \leq P$, then there exists an atom $G$ of $\mathscr{L}$ such that $G \leq P$ and $B_{i} \prec G$ for $i=1,2$.

Proof. Inductively define a sequence $G_{1}, \ldots, G_{k}$ of atoms of $\mathscr{L}$ as follows: let $G_{1}=A_{1}$ and assume for some $1 \leq m<k$ that $G_{m}$ has been defined so that

$$
G_{m} \leq \bigwedge_{i=1}^{m} L\left(A_{i}\right) \quad \text { and } \quad G_{m} \succeq B_{j} \quad j=1,2 .
$$

Since $G_{m} \succeq B_{1} \preceq A_{m+1} \succeq B_{2} \preceq G_{m}$ is a 4-cycle, there exists an atom $G_{m+1}$ of $\mathscr{L}$ such that

$$
G_{m+1} \preceq G_{m}, \quad G_{m+1} \preceq A_{m+1}, \quad \text { and } \quad G_{m+1} \succeq B_{i} \quad(i=1,2) .
$$

We clearly have $G_{m+1} \leq \bigwedge_{i=1}^{m+1} L\left(A_{i}\right)$, so the induction can proceed. Taking $G=G_{k}$ completes the proof.

LEMMA 13. Suppose that $\mathscr{L}$ is a CSL and that $A_{1}, \ldots, A_{n}$ are disjoint intervals of $\mathscr{L}$. Let $\mathscr{F}$ be the sublattice of $\mathscr{L}$ generated by $\left\{L\left(A_{1}\right), \ldots, L\left(A_{n}\right)\right\}$. For each atom $E$ of $\mathscr{F}$, let

$$
S(E)=\left\{i: L\left(A_{i}\right) E \neq 0\right\} \text {. }
$$


The following statements hold:

1. If $E$ is an atom of $\mathscr{F}$, then

$$
E=\left(\bigwedge_{i \in S(E)} L\left(A_{i}\right)\right)\left(\bigvee_{i \notin S(E)} L\left(A_{i}\right)\right)^{\perp}
$$

(We use the convention that $\bigwedge_{\text {empty set }} L\left(A_{i}\right)=I$ and $\bigvee_{\text {empty set }} L\left(A_{i}\right)$ $=0$.)

2. If $E$ and $F$ are atoms of $\mathscr{F}$, then

$$
E \preceq \mathscr{F} F \text { if and only if } S(F) \subseteq S(E) .
$$

Proof. Let $M$ be the set of all elements of $\mathscr{F}$ of the form

$$
\bigwedge_{i \in S} L\left(A_{i}\right)
$$

where $S$ is a subset of $\{1, \ldots, n\}$. Then the set $J$ of all joins of elements of $S$ is a distributive lattice which contains $L\left(A_{i}\right)$ for $1 \leq i \leq n$, and hence $J=\mathscr{F}$.

It is now easy to see that

$$
\bigwedge_{i \in S(E)} L\left(A_{i}\right)
$$

is the smallest element of $\mathscr{F}$ containing $E$ and

$$
\bigvee_{i \notin S(E)} L\left(A_{i}\right)
$$

is the largest element of $\mathscr{F}$ which does not contain $E$. The first statement follows.

Turning now to the second statement, let $P_{E}$ and $P_{F}$ be the smallest elements of $\mathscr{F}$ containing $E$ and $F$ respectively. If $E \preceq F$, then

$$
0 \neq E \mathscr{B}(\mathscr{H}) F=E \operatorname{Alg} \mathscr{F} F=E \operatorname{Alg} \mathscr{F} P_{F} F=P_{F} E \mathrm{Alg} \mathscr{F} F,
$$

and hence $P_{F} E \neq 0$. Therefore $S(F) \subseteq S(E)$.

Conversely, suppose that $S(F) \subseteq S(E)$. Then by the remarks in the second paragraph of this proof, we have $P_{E} \leq P_{F}$. Thus if $Q \in \mathscr{F}$ and $F \leq Q$, then $P_{E} \leq Q$. It follows that

$$
F \leq\left(\bigvee\left\{Q \in \mathscr{F}: Q \nsupseteq P_{E}\right\}\right)^{\perp} .
$$

By Lemma 23.3 of [11], if $x \in E \mathscr{H}$ and $y \in F \mathscr{H}$ are non-zero vectors, the rank one operator $x \otimes y^{*}$ belongs to $\operatorname{Alg} \mathscr{F}$. Hence $E \operatorname{Alg} \mathscr{F} F \neq(0)$. But then because $\mathscr{F}$ is a finite CSL, $E \operatorname{Alg} \mathscr{F} F=$ $E \mathscr{B}(\mathscr{H}) F$, whence $E \preceq F$. 
LEMMA 14. Let $\mathscr{L}$ be a purely atomic CSL which has the 4-cycle interpolation property. Let $\left\{A_{1}, \ldots, A_{n}\right\}$ be a finite collection of atoms of $\mathscr{L}$ and let $\mathscr{F}$ be the sub-CSL generated by $\left\{L\left(A_{i}\right)\right\}_{i=1}^{n}$. Let

$$
P=\bigvee_{i=1}^{n} L\left(A_{i}\right)
$$

and let $\chi$ be the set of all atoms $E$ of $\mathscr{F}$ such that $E \leq P$. Then there exists a function $E \mapsto B(E)$ from $\chi$ into the set of atoms of $\mathscr{L}$ such that

1. $B(E) \leq E$ for all $E \in \chi$ and

2. $E_{1} \preceq \mathscr{F} E_{2}$ if and only if $B\left(E_{1}\right) \preceq \mathscr{L} B\left(E_{2}\right)$ for all $E_{1}, E_{2} \in \chi$.

Proof. Given an atom $E$ of $\mathscr{F}$, define the order of $E$ to be the number

$$
\operatorname{ord}(E)=n-|S(E)|,
$$

where $S(E)$ is as in Lemma 13. Note that if $E_{1}$ and $E_{2}$ are atoms of $\mathscr{F}$ which have the same order, then $E_{1} \preceq E_{2}$ implies that $E_{1}=E_{2}$. For if $E_{1} \preceq E_{2}$, Lemma 13 implies that $S\left(E_{1}\right) \supseteq S\left(E_{2}\right)$; hence $S\left(E_{1}\right)=S\left(E_{2}\right)$ and the equality of the atoms follows from the first part of Lemma 13 .

Let $m_{1}<m_{2}<\cdots<m_{r}$ be a listing of the range of the restriction of the order function to the set $\chi$. Note that the atoms of order $m_{1}$ are the minimal atoms of $\mathscr{F}$ and also belong to $\mathscr{F}$. For $1 \leq t \leq r$, put

$$
\Omega_{t}=\left\{E \in \chi: \operatorname{ord}(E) \leq m_{t}\right\} .
$$

For each atom $E$ of $\mathscr{F}$ belonging to $\Omega_{1}$, let $B(E)$ be any atom of $\mathscr{L}$ contained in $E$. By our earlier remarks, we see that the atoms of order $m_{1}$ are all mutually incomparable (with respect to $\preceq_{\mathscr{F}}$ ). Thus, for all atoms $E_{1}$ and $E_{2}$ in $\Omega_{1}$, part (2) in the statement of the lemma holds.

Now suppose that for some integer $t$, we have defined $B(E)$ for all atoms of $\mathscr{F}$ belonging to $\Omega_{t}$ and that for all atoms $E_{1}$ and $E_{2}$ in $\Omega_{t}$, part (2) holds.

Fix an atom $F$ of $\mathscr{F}$ of order $m_{t+1}$, and fix any atom $B_{1}$ of $\mathscr{L}$ such that $B_{1} F=B_{1}$. Set

$$
\Delta=\left\{E \in \chi: \operatorname{ord}(E) \leq m_{t} \text { and } S(F) \subseteq S(E)\right\},
$$

and let

$$
P_{F}=\bigwedge_{i \in S(F)} L\left(A_{i}\right)
$$


By Lemma 13, $\Delta$ consists of the atoms $E$ of $\mathscr{F}$ such that $E \preceq \mathscr{F} F$.

Therefore, if $E \in \Delta, B(E) \leq P_{F}$. As $B_{1}$ is contained in $F$, we also have $B_{1} \leq P_{F}$. Apply Lemma 12 to conclude that for each $E \in \Delta$, there exists an atom $G(E)$ of $\mathscr{L}$ such that

$$
G(E) \leq P_{F} \quad \text { and } \quad B_{1} \preceq_{\mathscr{L}} G(E) \succeq_{\mathscr{L}} B(E) .
$$

We next show that there exists an atom $B(F)$ of $\mathscr{L}$ such that for all $E \in \Delta$,

$$
B(F) \leq P_{F} \quad \text { and } \quad B_{1} \preceq \mathscr{L} B(F) \succeq_{\mathscr{L}} G(E) .
$$

To do this, let $E_{1}, E_{2}, \ldots, E_{j}$ be a listing of the elements of $\Delta$. Let $C_{0}=B_{1}$. Since both $C_{0}$ and $G\left(E_{1}\right)$ are contained in $P_{F}$ we apply Lemma 12 to conclude that there exists an atom $C_{1}$ of $\mathscr{L}$ dominated by $P_{F}$ such that $C_{0} \preceq_{\mathscr{L}} C_{1} \succeq_{\mathscr{L}} G\left(E_{1}\right)$. Inductively repeat the procedure with the pair $C_{S-1}$ and $G\left(E_{S}\right)$ to obtain an atom $C_{s} \leq P$ of $\mathscr{L}$ such that

$$
C_{s-1} \preceq \mathscr{L} C_{s} \succeq_{\mathscr{L}} G\left(E_{s}\right) .
$$

Finally take $B(F)=C_{j}$.

We wish to prove that $B(F) F=B(F)$. Suppose that $H$ is the atom of $\mathscr{F}$ containing $B(F)$. Since

$$
B(F) \leq \bigwedge_{i \in S(F)} L\left(A_{i}\right)
$$

we see that $S(H) \supseteq S(F)$. Next, fix $j \in S(H)$. Since $L\left(A_{j}\right) H=H$, we have

$$
L\left(A_{j}\right) B(F)=B(F) .
$$

But $L\left(A_{j}\right) \in \mathscr{L}$ and $B_{1} \preceq \mathscr{L} B(F)$, so $L\left(A_{j}\right) B_{1}=B_{1}$. However, $L\left(A_{j}\right)$ also belongs to $\mathscr{F}$ and as $B_{1} \leq F$, we have $L\left(A_{j}\right) F=F$. Therefore, $S(H) \subseteq S(F)$, so $S(H)=S(F)$. By Lemma 13 we have $H=F$. We have proved that $F$ dominates $B(F)$.

Next, if $E \in \Omega_{t}$ and $E \preceq \mathscr{F} F$, then $E \in \Delta$. Then by (6) and (7),

$$
B(E) \preceq \mathscr{L} B(F) .
$$

On the other hand, if $E \in \Omega_{t}$, and $B(E) \preceq \mathscr{L} B(F)$, then for any $k \in S(F)$,

$$
B(F) L\left(A_{k}\right)=B(F) \text { and hence } B(E) L\left(A_{k}\right)=B(E) .
$$

It follows that $S(F) \subseteq S(E)$, and therefore $E \preceq \mathscr{F} F$. 
The previous paragraph, together with the fact that distinct atoms of the same order are not comparable, shows that for any $E \in \Omega_{t+1}$,

$$
B(F) \succeq_{\mathscr{L}} B(E) \text { if and only if } F \succeq_{\mathscr{F}} E .
$$

We have now defined $B(F)$ for all $F \in \Omega_{t+1}$ satisfying both parts of the statement of the lemma.

Indication now completes the proof.

Proposition 15. Suppose that $\mathscr{L}$ is a purely atomic CSL which has the 4-cycle interpolation property. Then $\mathscr{L}$ has the compression property.

Proof. Let $\Lambda$ be the collection of all finite sets whose elements are atoms of $\mathscr{L}$, and direct $\Lambda$ by inclusion. For each $\lambda \in \Lambda$, let $\mathscr{F}_{\lambda}$ be the sublattice of $\mathscr{L}$ generated by $\{L(A): A \in \lambda\}$, let

$$
P_{\lambda}=\bigvee_{A \in \lambda} L(A)
$$

and let $\chi_{\lambda}$ be the set of all atoms of $\mathscr{F}_{\lambda}$ dominated by $P_{\lambda}$.

Clearly $\cup_{\lambda} \mathscr{F}_{\lambda}$ is strongly dense in $\mathscr{L}$.

The preceding lemma shows that if

$$
R_{\lambda}=\sum_{E \in \chi_{\lambda}} B(E)
$$

then $R_{\lambda} \mathscr{L}=R_{\lambda} \mathscr{F}$. Also, we defined the mapping $E \mapsto B(E)$ for all atoms of $\mathscr{F}_{\lambda}$ except perhaps for the atoms whose order is the cardinality of $\lambda$. Hence $\mathscr{F}_{\lambda}$ is order isomorphic either to $R_{\lambda} \mathscr{L}$ or to $\mathscr{C}\left(R_{\lambda} \mathscr{L}\right)$.

We now have:

THEOREM 16. If $\mathscr{L}$ is a purely atomic, hyperreflexive, infinite multiplicity CSL with the 4-cycle interpolation property, then $\operatorname{Alg} \mathscr{L}$ has the perturbation property.

Proof. Combine Propositions 11 and 15.

Question. Is it possible to remove the hypothesis that $\mathscr{L}$ satisfies the 4-cycle interpolation condition in the statement of Theorem 16? This would be possible if we knew that all purely atomic CSLs have the compression property. 
Notes added in proof. Davidson has pointed out to the author that Proposition 10 can be proved without using Theorem 3 .

The author has recently proved (see [24]) that if $\mathscr{L}$ and $\mathscr{M}$ are any CSLs such that $d(\operatorname{Alg} \mathscr{L}, \operatorname{Alg} \mathscr{M})=\delta<\frac{1}{36}$, then there exists $S \in \mathscr{B}(\mathscr{H})$ such that $\|S-I\|<12 \delta$ and $S(\operatorname{Alg} \mathscr{L}) S^{-1}=\operatorname{Alg} \mathscr{M}$. This result gives an alternate approach to Theorem 3 and improves Theorem 16.

\section{REFERENCES}

[1] W. B. Arveson, Operator algebras and invariant subspaces, Ann. Math., 100 (3) (1974), 433-532.

[2] M. D. Choi and K. Davidson, Perturbations of matrix algebras, Michigan Math. J., 33 (1986), 273-287.

[3] E. Christensen, Perturbations of operator algebras, Invent. Math., 43 (1977), 1-13.

[4] _ Perturbations of operator algebras II, Indiana Univ. Math. J., 26 (1977), 891-904.

[5] _ Perturbations of type I von Neumann algebras, J. London Math. Soc., 9 (1975), 395-405.

[6] K. R. Davidson, Perturbations of reflexive operator algebras, J. Operator Theory, 15 (1986), 289-305.

[7] _ When locally contractive representations are completely contractive, preprint, 1992.

[8] K. R. Davidson and M. S. Ordower, Some exact distance constants, preprint, 1992.

[9] K. R. Davidson and D. R. Pitts, Approximate unitary equivalence of completely distributive commutative subspace lattices, preprint, 1993.

[10] K. R. Davidson and S. Power, Failure of the distance formula, J. London Math. Soc. (2), 32 (1984), 157-165.

[11] K. R. Davidson, Nest Algebras, volume 191 of Research Notes in Math., Pitman, Boston-London-Melbourne, 1988.

[12] K. R. Davidson, V. I. Paulsen and S. C. Power, Tree algebras, semidiscreteness, and dilation theory, J. London Math. Soc., to appear.

[13] F. Gilfeather and R. L. Moore, Isomorphisms of certain CSL algebras, J. Funct. Anal., 67 (1986), 264-291.

[14] F. Gilfeather and R. R. Smith, Cohomology for operator algebras: cones and suspensions, J. London Math. Soc., to appear.

[15] F. Gilfeather, A. Hopenwasser and D. Larson, Reflexive algebras with finite width lattices: tensor products, cohomology, compact perturbations, J. Funct. Anal., 55 (1984), 175-199.

[16] B. E. Johnson, Perturbations of Banach algebras, Proc. London Math. Soc. (3), 34 (1977), 439-458.

[17] B. Johnson, R. Kadison and J. Ringrose, Cohomology of operator algebras III: reduction to normal cohomology, Bull. Soc. Math. France, 100 (1972), 73-96.

[18] R. V. Kadison and D. Kastler, Perturbations of von Neumann algebras I: stability of type, Amer. J. Math., 94 (1972), 38-54. 
[19] E. C. Lance, Cohomology and perturbations of nest algebras, Proc. London Math. Soc., 43 (1981), 334-356.

[20] D. R. Larson, Triangularity in operator algebras, in Surveys of some recent results in operator theory, Vol. II, Pitman Research Notes in Math., No. 192, Longman Scientific and Technical, Harlow, 1988, pages 121-188.

[21] I. Raeburn and J. Taylor, Hochschild cohomology and perturbations of Banach algebras, J. Funct. Anal., 25 (1977), 258-266.

[22] S. Rosenoer, Distance estimates for von Neumann algebras, Proc. Amer. Math. Soc., 86 (1982), 248-252.

[23] J. Wermer, Commuting spectral operators on Hilbert space, Pacific J. Math., 4 (1954), 355-361.

[24] D. R. Pitts, Close CSL algebras are similar, preprint, 1993.

Received May 16, 1992 and in revised form February 25, 1993. Research partially supported by an NSF Postdoctoral Fellowship and by NSF grant DMS-9204811.

UNIVERSITY OF NEBRASKA

LINCOLN, NE 68588-0323 


\section{PACIFIC JOURNAL OF MATHEMATICS}

Founded by

E. F. BECKENBACH (1906-1982) F. Wolf (1904-1989)

\section{EDITORS}

Sun-Yung A. Chang

(Managing Editor)

University of California

Los Angeles, CA 90024-1555

chang@math.ucla.edu

\section{F. Michael Christ}

University of California

Los Angeles, CA 90024-1555

christ@math.ucla.edu

Herbert Clemens

University of Utah

Salt Lake City, UT 84112

clemens@math.utah.edu

\author{
ThOMAs ENRIGHT \\ University of California, San Diego \\ La Jolla, CA 92093 \\ tenright@ucsd.edu \\ Nicholas ERcolaNI \\ University of Arizona \\ Tucson, AZ 85721 \\ ercolani@math.arizona.edu \\ R. FINN \\ Stanford University \\ Stanford, CA 94305 \\ finn@gauss.stanford.edu \\ VAUGHAN F. R. Jones \\ University of California \\ Berkeley, CA 94720 \\ vfr@math.berkeley.edu
}

\author{
STEVEn KerCKHOFF \\ Stanford University \\ Stanford, CA 94305 \\ spk@gauss.stanford.edu
}

Martin ScharlemanN

University of California

Santa Barbara, CA 93106

mgscharl@math.ucsb.edu

\author{
Harold Stark \\ University of California, San Diego \\ La Jolla, CA 92093 \\ V. S. VARADARAJAN \\ University of California \\ Los Angeles, CA 90024-1555 \\ vsv@math.ucla.edu
}

\section{SUPPORTING INSTITUTIONS}

UNIVERSITY OF ARIZONA

UNIVERSITY OF BRITISH COLUMBIA

CALIFORNIA INSTITUTE OF TECHNOLOGY

UNIVERSITY OF CALIFORNIA

UNIVERSITY OF MONTANA

UNIVERSITY OF NEVADA, RENO

NEW MEXICO STATE UNIVERSITY

OREGON STATE UNIVERSITY

\author{
UNIVERSITY OF OREGON \\ UNIVERSITY OF SOUTHERN CALIFORNIA \\ STANFORD UNIVERSITY \\ UNIVERSITY OF HAWAII \\ UNIVERSITY OF UTAH \\ WASHINGTON STATE UNIVERSITY \\ UNIVERSITY OF WASHINGTON
}

The Supporting Institutions listed above contribute to the cost of publication of this Journal, but they are not owners or publishers and have no responsibility for its content or policies.

Mathematical papers intended for publication in the Pacific Journal of Mathematics should be in typed form or offset-reproduced (not dittoed), double spaced with large margins. Please do not use built up fractions in the text of the manuscript. However, you may use them in the displayed equations. Underline Greek letters in red, German in green, and script in blue. The first paragraph must be capable of being used separately as a synopsis of the entire paper. In particular it should contain no bibliographic references. Please propose a heading for the odd numbered pages of less than 35 characters. Manuscripts, in triplicate, may be sent to any one of the editors. Please classify according to the 1991 Mathematics Subject Classification scheme which can be found in the December index volumes of Mathematical Reviews. Supply name and address of author to whom proofs should be sent. All other communications should be addressed to the managing editor, or Julie Honig, University of California, Los Angeles, California 90024-1555.

There are page-charges associated with articles appearing in the Pacific Journal of Mathematics. These charges are expected to be paid by the author's University, Government Agency or Company. If the author or authors do not have access to such Institutional support these charges are waived. Single authors will receive 75 free reprints; joint authors will receive a total of 100 free reprints. Additional copies may be obtained at cost in multiples of 50 .

The Pacific Journal of Mathematics (ISSN 0030-8730) is published monthly except for July and August. Regular subscription rate: $\$ 215.00$ a year (10 issues). Special rate: $\$ 108.00$ a year to individual members of supporting institutions.

Subscriptions, orders for numbers issued in the last three calendar years, and changes of address should be sent to Pacific Journal of Mathematics, P.O. Box 4163, Berkeley, CA 94704-0163, U.S.A. Old back numbers obtainable from Kraus Periodicals Co., Route 100, Millwood, NY 10546.

The Pacific Journal of Mathematics at University of California, c/o Department of Mathematics, 981 Evans Hall, Berkeley, CA 94720 (ISSN 0030-8730) is published monthly except for July and August. Second-class postage paid at Berkeley, CA 94704, and additional mailing offices. POSTMASTER: send address changes to Pacific Journal of Mathematics, P.O. Box 4163, Berkeley, CA 94704-0163.

PUBLISHED BY PACIFIC JOURNAL OF MATHEMATICS at University of California, Berkeley, CA 94720, A NON-PROFIT CORPORATION

This publication was typeset using $\mathcal{A} \mathcal{M} \mathcal{S}-\mathrm{T}_{\mathrm{E}} \mathrm{X}$,

the American Mathematical Society's $T_{E} X$ macro system.

Copyright (c) 1994 by Pacific Journal of Mathematics 


\section{PACIFIC JOURNAL OF MATHEMATICS}

Volume $165 \quad$ No. $1 \quad$ September 1994

The effect of dimension on certain geometric problems of irregularities of 1 distribution

RALPH ALEXANDER

The structure of $\mathrm{sl}(2,1)$-supersymmetry: irreducible representations and primitive ideals

Didier ARnAL, HÉdi BENAMOR and GEORges PinCZON

Periods and Lefschetz zeta functions

Josefina CASASAYas, JaUme Llibre and ANA Nunes

On closed hypersurfaces of constant scalar curvatures and mean curvatures in 67 $S^{n+1}$

\section{SHAOPING CHANG}

$R$-groups and elliptic representations for $\mathrm{SL}_{n}$

DAVID GOLDBERG

The boundary distortion of a quasiconformal mapping

JuHA HeINONEN and PEKKA KosKela

Strongly approximately transitive group actions, the Choquet-Deny theorem, 115 and polynomial growth

\section{WOJCIECH JAWORSKI}

$q$-canonical commutation relations and stability of the Cuntz algebra

PALle E. T. Jorgensen, L. M. Schmitt and Reinhard Frank

WERNER

Complete open manifolds of non-negative radial curvature

YOSHIROH MACHIGASHIRA

Perturbations of certain reflexive algebras

DAVID RYDER PITTS

Interpolation submanifolds of the unitary group 